Commentary on: Telephone-Based, Clinician-Guided Self-Help Cognitive Behavioral Therapy for Depression in Parkinson's Disease (dPD): The Responder Cases of "Alice" and "Carl," and the Nonresponder Cases of "Ethan" and "Gary"

\title{
Telemental Health During a Global Pandemic: Clinical Lessons from Guided Self-Help, Telephone Therapy Case Studies
}

\author{
LIZA E. PINCUS ${ }^{a, b}$ \\ ${ }^{\text {a }}$ Graduate School of Applied \& Professional Psychology, Rutgers-New Brunswick, NJ \\ b Correspondence regarding this article should be sent to: Liza Pincus, Rutgers University, 152 Frelinghuysen Road, \\ Busch Campus, Piscataway, NJ 08854 \\ Email: liza.pincus@rutgers.edu
}

\begin{abstract}
I am a fourth year clinical psychology doctoral student at the Graduate School of Applied and Professional Psychology (GSAPP) at Rutgers University. I have devoted much of my graduate training to exploring effective tools for the dissemination and implementation of mental health services, with telehealth chief among them. However, until the COVID-19 pandemic hit, I had had no personal experience delivering psychotherapy treatment via virtual platforms. Reviewing Dr. Logan Durland's (2020) lessons from treating Parkinson's disease patients via telephone therapy has served as an excellent guide for navigating treatment, both during the pandemic and in the future.
\end{abstract}

Key words: Parkinson's disease (PD); depression in Parkinson's disease (dPD); telehealth; telemental health; cognitive behavioral therapy (CBT); manualized therapy; case studies; clinical case studies

\section{INTRODUCTION}

There is likely no better time to review a manuscript about telemental ${ }^{1}$ health than during a global pandemic.

I am a fourth-year clinical psychology doctoral student and I have focused my research on increasing access to mental healthcare through the dissemination and implementation of evidence-based treatment. Programs such as "Telehealth Guided Self-Help for Depression in

\footnotetext{
${ }^{1}$ In this commentary I interchangeably use the term "telehealth" services, which refers to the use of telecommunications technology in providing health services generally; the term "telemental" services, which refers to telehealth services as they specifically apply to mental health services; and the term "teletherapy," which refers to telemental services that consist of distance psychotherapy.
} 
Parkinson's Disease" (“TH-GSH-dPD”), as laid out in Dr. Logan Durland's case study, have enormous potential to close the "treatment gap" in both global and national mental healthcare.

The "treatment gap" - the percentage of the population that needs mental health services but can or does not access them - has been found to exceed 50\% in all countries, and approach $90 \%$ in the least resourced countries (Patel et al., 2010). As Durland and others have suggested, telehealth breaks down many of the most common barriers to receiving treatment. For one, there is dearth of trained mental health professionals in many parts of the world, including in the United States. A 2007 study found that there were only approximately 16 psychologists per 100,000 residents in rural areas across the country (APA, 2007). Telehealth not only removes the need for patients to live in close proximity to their therapists, but also lowers barriers to treatment such as transportation, overall cost, and potential stigma of in-person visits. Finally, and most relevant to Durland's case study, telemental health allows chronically ill patients to access treatment without having to leave home (Durland, p. 12).

Until two months ago, my experience with telemental health had been entirely hypothetical. I championed the use of technology to reduce the common treatment barriers discussed above. Crucially, I also only understood some of the most common barriers to the widespread adoption of telemental health - namely clinician and patient preference related to such factors as therapeutic alliance (Cowan et al., 2019) — in theoretical terms.

However, my experience with telemental health suddenly became personal when the impact of COVID-19 forced much of the country, including most outpatient clinicians like myself to go into lockdown in mid-March of this year. I began to experience firsthand both the benefits and challenges of telehealth. For example, I immediately saw session attendance increase, as barriers to treatment were effectively eroded for patients with secure, reliable Internet access. On the other hand, having been trained entirely for in-person therapy, I instantly struggled to maintain rapport with patients over a virtual therapy platform. And, as in many other areas of society, the pandemic has exposed inequalities that have been present all along and that interfere with effective telehealth delivery. For example, some patients do not have private spaces at home to engage in therapy, and some do not have reliable Internet connections.

If the field of mental healthcare is going to continue moving in the direction of telehealth, which seems inevitable in the post-pandemic world, we would be wise to consider the clinical implications in order to inform existing practices as well as future clinical training. While numerous randomized control trials have demonstrated the efficacy and effectiveness of specific teletherapy interventions, and many articles have provided logistical guidance on how to execute such interventions, Durland's case studies provide a unique perspective on conducting teletherapy with a particular dPD patient, within the complex contexts of their personality, their cognitive ability, their physical health condition, their life circumstances, and their relationship with their caregiver. The lessons Durland lays out in his four case studies serve as clinical guidance for the many clinicians currently entering a new frontier of online therapy.

I will focus in my commentary on a few themes in Durland's case studies that seem particularly relevant for clinicians, like me, who are conducting telemental health for the first time. These themes include: selecting the appropriate platform (telephone versus 
videoconference); incorporating caregiver and/or family member involvement; and adapting treatment for online platforms. Throughout the commentary, I will consider the therapeutic alliance within the context of teletherapy.

\section{TELEPHONE VERSUS VIDEOCONFERENCE}

Durland discusses at length the ways in which the telephone format led to clinical challenges, and may have even contributed to the two "nonresponsive" treatment outcomes. He lists telephone connection issues (p. 36) and difficulty deciphering speech over the telephone (p. 110 ) as treatment-interfering factors. He discusses the absence of visual cues contributing to an inability to manage one patient's "tangentiality" (p. 72), thereby interrupting the session agenda and limiting overall progress toward treatment goals. Similarly, Durland cites the telephone format as interfering with the therapeutic alliance, citing difficulty conveying empathy toward the patient without the use of body language and facial expression (p. 133).

In his discussion of these drawbacks of telephone therapy as they potentially interfere with both treatment progress and the therapeutic alliance, Durland compares telephone therapy with in-person therapy (p. 133). He suggests that the in-person format may be better suited for some patients who tend to be more "tangential" (as in the case of "Carl"), or patients who require visual cues to convey empathy (as in the case of "Gary"). However, in reading the specific limitations of telephone therapy Durland outlines, it appears some of them could potentially be addressed by using a videoconferencing platform instead of the telephone. The opportunity for the clinician and patient to see one another's faces during therapy would re-introduce visual cues to re-direct "tangentiality," as well as to convey empathy through facial expression. For example, some videoconferencing platforms offer virtual "whiteboards," enabling clinicians to write out session agendas and reference them during sessions.

And yet, while Durland conducted therapy within the context of a research trial and was therefore not authorized to switch telehealth platforms, his case studies provide guidance on the advantages of choosing telephone therapy over videoconferencing. For one, older adults, the patient population in the "TH-GSH-dPD" study, may be better candidates for telephone therapy because they may have less familiarity with and access to videoconferencing (Brenes et al., 2015).

Furthermore, Durland often mentions throughout his manuscript that the depression in Parkinson's disease (dPD) patients experienced shame and anxiety related to the physical effects of Parkinson's disease, namely tremors. Consistent with the finding that telephone therapy is effective for patients with co-occurring medical disorders (Heckman et al., 2017), it may be therapeutically advantageous for patients with physical limitations to speak unselfconsciously over the telephone, where their physical movements remain unseen. The notion of the "online disinhibition effect," whereby patients may be more open to freely disclosing their problems and emotions when speaking on the telephone, has been demonstrated in the telehealth literature (Suler, 2004). 
Finally, telephone therapy has additional advantages in terms of treatment access over videoconferencing. While perhaps less relevant to Durland's case studies because of the caregiver involvement in treatment, mobile phones are more easily transportable than computers, such that a patient could sit in a car or take a walk while talking to their therapist, if they do not have privacy at home. Furthermore, many underserved populations do not have access to video chat or high-speed Internet (APA, 2020).

\section{CAREGIVER INVOLVEMENT}

One of the unique aspects of "TH-GSH-dPD" as a treatment protocol is its emphasis on caregiver involvement in therapy. Among the treatment "responders," the support and reinforcement of their caregivers appears to have contributed significantly to treatment gains.

Durland notes several clinical advantages of repeated caregiver involvement in treatment. In general, in the three cases where caregivers actively participated, Durland as the therapist benefitted from an additional marker of treatment progress, through the caregivers' reports. In the case of "Alice," Durland used a conflict between his patient and her spouse as an opportunity to intervene and model more effective communication. Durland thereby improved not only a specific treatment outcome for his patient, but also the relationship between the patient and her spouse (p. 36). Durland's successful modeling is particularly impressive given that he delivered it via telephone, and demonstrates that such interventions can be done through telehealth.

In the case of "Ethan" and his caregiver "Fay," Durland observed ways in which Fay contributed to an "undoing" of treatment gains through unfavorable behavioral reinforcement related to Ethan. However, I would suspect that even in this case, it was still clinically useful to engage Fay in Ethan's treatment. Because a caregiver is such a crucial role in an individual's life, it is likely that Fay's behavior would have served as a barrier to Ethan's treatment response, whether or not Durland as the therapist was acutely aware of the issue. At least Durland had the opportunity to address his concerns with both Fay and Ethan in therapy, as opposed to treatment gains being "undone" by a family member outside of the therapist's awareness, as I sometimes suspect is the case in therapy.

Caregiver involvement in therapy is a crucial advantage of teletherapy over in-person treatment, and should be given special consideration during the pandemic, when the majority of formerly in-person therapy is now taking place in patients' homes. Teletherapy provides a unique opportunity to engage caregivers, parents, and partners in a way that is often challenging to achieve in-person, due to practical limitations such as work and childcare, and barriers such as mental health stigma.

\section{TREATMENT ADAPTATIONS}

Using a "Guided Self-Help" (GSH) format to deliver the depression in Parkinson's disease treatment makes particular sense in the context of increasing access to care and providing written materials to supplement learning among Parkinson's patients (p. 13). And yet, as is the 
case with all evidence-based therapies, Durland discovered several ways in which certain patients did not optimally respond to the specific materials in the GSH protocol.

One of the strongest examples of adapting a manualized treatment to fit the needs of the individual patient occurred in Durland's treatment with Ethan, for whom English was a second language and who suffered from memory problems related to Parkinson's disease. Durland learned through his struggle to successfully implement the GSH protocol exactly-as-written that changing some language was crucial for treatment acceptability in Ethan's case (p. 111). While Durland ultimately adapted the language in the treatment protocol so that Ethan could better understand the concepts, perhaps this process could have been expedited if Ethan and his caretaker wife Fay had been able to utilize visual cues and tools, either through in-person or video therapy. It is likely that Durland would have picked up on Ethan's lack of understanding more quickly had he had been able to see Ethan's facial expressions. Durland could also have used a physical or digital whiteboard to write or draw out concepts.

I was particularly impressed by Durland's self-reflection following his work with "Gary," whose treatment "nonresponse" appears to have been closely tied to the treatment structure and format. Gary experienced cognitive restructuring, one of the primary mechanisms of the "THGSP-dPD" protocol, as invalidating (p. 134). Durland reflects that Gary's difficulty accepting his Parkinson's diagnosis made the idea of changing his thoughts and behaviors associated with that diagnosis very challenging (p. 135). Durland suggests potential ways that being more flexible within the context of a manualized treatment could have helped improve treatment outcomes: perhaps they could have begun the treatment with Motivational Interviewing to increase Gary's commitment to treatment, and/or first worked on accepting his diagnosis before trying to change his behavior (p. 137). Finally, Durland notes that Gary reported complaints with the telephone format, stating that it interfered with their "one-on-one connection," and discusses ways in which he worked to build their rapport over the telephone. He also wonders if Gary may have benefited more from conducing treatment via an alternative platform (p. 136).

The ways in which Durland both adapted "TH-GSP-dPD" and recommends that other clinicians adapt it in the future, speak to the importance of flexibility in delivering any manualized treatment. This is true regardless of treatment platform, but is especially important when delivering therapy remotely. It could be that if this had not been a randomized control trial and Durland and Gary had tried videoconferencing, Gary may have felt less distracted, thus improving their therapeutic relationship. It is also possible that Gary's therapy-interfering behaviors would have been better addressed in person, as long as he did not face significant barriers to in-person care.

When addressing the global treatment gap in mental healthcare, there is a need to balance pragmatism with the individual, clinical needs of patients. The COVID-19 pandemic is certainly an extreme example of needing to make do with telehealth platforms, regardless of whether inperson therapy is preferable or even clinically indicated for an individual. However, postpandemic, clinicians and patients will once again have to consider barriers to in-person treatment alongside clinical indications. Making treatment adaptations to teletherapies, when possible given access to technology, is one way to address individual, clinical needs. Another approach is 
to consider a "stepped care" approach to treatment, similar to the medical model, in which all individuals receive a lower-intensity, perhaps online treatment, and then advance to more levels of care, perhaps transitioning to in-person care, if needed (Patel, 2017).

\section{CONCLUSION}

Durland's four case studies on cognitive-behavioral treatment for depression in Parkinson's disease, delivered via telehealth, provide important lessons at a moment when most clinicians are being forced by external circumstances to adapt to a new way of working. Durland offers guidance related to pros and cons of telephone therapy versus videoconferencing, and caregiver involvement in and treatment adaptations to teletherapy. Ultimately, while many clinicians and patients fear the loss of a therapeutic alliance in the context of telehealth, Durland's work proves that aspects of telehealth can actually improve treatment outcomes. It also proves that treatment flexibility can ameliorate what is currently a necessary aspect of therapy. Flexibility in delivering treatment remotely is crucial, given the potential of telehealth to continue to expand treatment access.

\section{REFERENCES}

American Psychological Association. (2007). Statement on services by telephone, teleconferencing, and internet. Retrieved on May 12, 2020, from https://www.apa.org/ethics/education/telephone-statement.aspx.

American Psychological Association. (2020). Telephone psychotherapy: ensuring patients have access to effective care. Retrieved on May 12, 2020 from https://www.apaservices.org/practice/legal/technology/telephone-psychotherapy.aspx.

Brenes, G. A., Danhauer, S. C., Lyles, M. F., Hogan, P., \& Miller, M. E. (2015). Telephonedelivered cognitive behavioral therapy and telephone-delivered nondirective supportive therapy for rural older adults with generalized anxiety disorder. JAMA Psychiatry, 72(10): 1012-1020.

Cowan, K. E., McKean, A. J., Gentry, M. T., Hilty, D. M. (2019). Barriers to use of telepsychiatry: clinicians as gatekeepers. Mayo Clinic, 94(12): 2510-2523.

Durland, L. (2020). Telephone-based, clinician-guided self-help cognitive behavioral therapy for depression in Parkinson's disease (dPD): The responder cases of "Alice" and "Carl," and the nonresponder cases of "Ethan" and "Gary." Pragmatic Case Studies in Psychotherapy, 16(1), Article 1, 1-103. Available: http://pcsp.libraries.rutgers.edu/

Heckman, T. G., Heckman, B. D., Anderson, T., Lovejoy, T. I., Markowitz, J. C., Shen, Y., \& Sutton, M. (2017). Tele-interpersonal psychotherapy acutely reduces depressive symptoms in depressed HIV-infected rural persons: a randomized clinical trial. Behavioral Medicine, 43(4): 285-295.

Patel, V. (2017). Talking sensibly about depression. PLoS Medicine, 14(4): e1002257.

Patel, V., Maj, M., Flisher, A. J., De Silva, M. J., Koschorke, M., \& Prince, M. (2010). Reducing the treatment gap for mental disorders: a WPA survey. World Psychiatry, 9(3): 169-176.

Suler, J. (2004). The online disinhibition effect. Cyberpsychology \& Behavior, 7(3), 321-326. 